night after operation it was noted that the turgidity of the fundus veins was. less.

For about three months after operation there were occasional attacks of pain round the right eye, but these gradually ceased. When last seen (Dec., 1925) the retinal vessels were of not more than normal size; the depth of the cup was $5.5 \mathrm{D}$. to $6 \mathrm{D}$.; central vision was $6 / 6$ (partly); the area of the field of vision was the same as before. A feature of the case which was not obviously explicable was that the right eye was slightly $\left({ }^{\prime \prime}\right)$ more prominent than the left. It remained so after operation.

No history could be obtained which enabled one to date the onset of the glaucoma. The outgrowth of uvea through the pupil suggested that it had been present for at least a couple of years. It seems possible that it had something to do with the onset of puberty.

\title{
BILATERAL CYSTS OF THE CORNEA
}

BY

L. Webster Fox, M.D., LL.D.

PHILADELPHIA

CySTs of the cornea must be very rare indeed, since a careful search of the tabulated and indexed records in ophthalmology brings to our attention only the case reported by de Schweinitz and Crampton in the Ophthalmic Record, Vol. XX, page 42, 1911. There are other case reports of cysts of the cornea secondary to direct injury recorded by Collins, Schieck, Czermak, Gruening, Tertsch, Augstein, Clausnizer and Tirumurti, but only that reported by the first-mentioned observer corresponds with the case here under observation. Even including all of these cases the condition is by no means commonplace. In the American Journal of Ophthalmology among the abstracts there is a note upon this subject by Fileti, published in the Ann. di Ottal. e Clin. Ocul., Vol. 53, p. 696, 1925, concerning the bibliography of the condition. According to this writer only twenty cases are reported in literature and of these only eleven have included pathological findings. Also in this paper he describes a case seen by himself in which the condition followed an inflammatory affection of the cornea. He excised the cyst in one instance, but was unable to demonstrate any connection with the anterior chamber. The vision was reduced to $1 / 60$ in one eye and $1 / 14$ in the other and was not improved by operation. These cysts were true cysts and lined with endothelium. The cyst in our case was in reality a separation of the lamellae of the cornea. 
The patient in this instance was a little Jewish girl about eleven years of age who was the subject of a pronounced convergent squint when she was about five years old. She had come under the writer's observation at that time, but the parents tired of the routine of training, and in the early part of 1925, sought more radical relief from other sources. At this time she underwent a double advancement operation at the hands of another surgeon. Heapings up of the conjunctiva had marked the sites of the anchorages of the advancements and alarmed the parents. They were assured by the operator that these would soon subside, which they seemed to do, but not very rapidly. The squint was overcome however, but only for a short time, after which the eyes returned to their former alignment. Three months prior to her return to our service the mother had noticed white spots appearing upon the cornea, most pronounced in the left eye. She returned to the surgeon who had performed the advancement but was assured they were of no moment and would soon disappear, according to the mother's statement.

The patient was then taken to the Wills Eye Hospital in this city where she was advised that an operation would be necessary, but what kind or for which of her several eye conditions, the mother did not seem to understand. This was in November, 1925, and the record at Wills Eye Hospital showed that her vision was 6/60 and $6 / 12$, and that no view of her eyegrounds was obtained on account of the severe blepharospasm. The report furnished us was vague as to the other conditions. At the time she presented herself for our second observation several striking features were noted. In the first place the squint had failed of complete relief and considerable convergent strabismus was still present. At about the site of the anchorage of the advancement in the left eye, a V-shaped separation of the sclera was to be seen, in the bottom of which was the dark blue pigment of the choroidal coat. This V-shaped separation extended into the cornea where the diffraction resulted in giving it a steam-like aspect. In the right eye it was possible to demonstrate a very much less marked condition of the same character.

The importance of this case justified us in making an intensive study of it from all angles. An attempt was made to study the case with the slit-lamp without having the pupils dilated, and while much information was obtained thereby, subsequent examinations with the pupils dilated were more satisfactory. The right eye did not show at all well without a mydriatic. The illumination was too strong and passed right through the steamy area. In the left eye the corneal segment made by the narrow beam of the slitlamp passed over the $\mathrm{V}$-shaped steamy area, and shortly became two- one with the convexity anteriorly and the other with. the 
convexity posteriorly--showing that here was a cyst, and that these images were due to the anterior and posterior walls of the cavity respectively. As the apex of the steamy area was reached, which was about the centre of the cornea the images approached each other fusing as the healthy normal cornea was reached. The infiltration seemed to be beneath Bowman's membrane anteriorly and in front of Descemet's membrane posteriorly. Presumably this connected with the vitreous, or at least with the sub-retinal space, through the whole vitreous. This explanation is necessarily speculative.

A subsequent examination by the slit-lamp with the pupils dilated showed a confirmation of the previous findings but demonstrated in addition the presence of blood-vessels in the limbs of the Vshaped steamy opacity of the cornea of the left eye. The perforation of the sclera was more easily made out at this examination and the details readily discerned. Altogether the condition appeared to me more marked throughout than upon the previous examination. The limbs of the $\mathrm{V}$ were becoming wider apart and the apex seemed to be progressing across the cornea.

This patient was most carefully examined and studied upon several occasions in order to demonstrate beyond peradventure of a doubt that the middle layers of the cornea had been split, and that fluid, possibly from the interior of the eye, now occupied the cavity produced by the splitting.

The progressive character of the condition prompted us to undertake a surgical operation. Accordingly after proper preparation the patient was etherized. After the eye speculum had been introduced in the left eye the globe was grasped and held firmly by fixation forceps and a smaller angular keratome was passed into the cornea at the upper margin on the temporal side of the steamy area. The point of the instrument was then turned down so that it re-entered the cornea from above and opened the cystic cavity vertically. A considerable amount of fluid escaped, some of which must have been aqueous since the keratome must have entered the anterior chamber on its initial passage through the cornea. The Stevens' scissors were then introduced into the wound and made to cut through the lower margin of the steamy V. This had the effect of cutting off the blood supply, which the corneal microscope had shown us to be quite profuse. The entire cystic area collapsed and the cotnea and adjacent tissue became flat, thereby justifying our diagnosis and treatment. The edges of the corneal wound were cauterized with a weak solution (one per cent.) of trichloracetic acid, and novoform-atropine ointment was applied followed by the usual aseptic dressing.

Upon examination with the slit-lamp (following an uninterrupted convalescence) about two weeks after the operation the eye appeared 
to be quiet. The hernia of the ciliary body previously noted had completely subsided and the scleral opening appeared to have closed. The cornea was remarkably clear, and a few active vessels could be seen above, although these had been severed in the operation, and were expected to have collapsed. The corneal opacity-the steamy portion-seemed to have dropped slightly below the pupillary area, and did not occupy the central portion as formerly. There was no bulging. The iris was now easily visible through all portions of the area previously occupied by the steamy opacity. As the beam of the slit-lamp was passed over from the normal cornea to the site of the cystic cornea, it underwent distortion, but maintained its singular identity. Previously, it will be recalled, it divided into two beams, one with the convexity anteriorly and the other with its convexity posteriorly.

Ophthalmological examination upon March 30, 1926, showed the vision of the right eye (the unoperated eye) to be $5 / 60$, both with and without glasses, but in the left eye (the operated eye) it was $5 / 6$ without glasses and $5 / 5$ with glasses. Both eyes were quiet. The hernia of the choroid previously noted in the left eye was no longer in evidence. There was a very small vesicle at the site of the corneal incision above and a few vessels could be made out, but the walls of the cyst remained in close contact and only one beam could be demonstrated by the slit-lamp. The result was highly satisfactory to the patient and her mother as well as to the operator.

Examination upon June 30, 1926, showed the following details. The right eye showed the cornea to be entirely clear-no opacity was visible as was noted upon the first examination. The eye was slightly convergent. There was still considerable heaping up of the conjunctiva and episcleral tissue at the site of the advancement anchorage. There was in the midst of this a pin-head sized bluishblack area presumably due to a hernia of some part of the uveal tract, perhaps the choroid or ciliary body. The vision was $6 / 45$ and with lenses could be brought to $6 / 12$, and at close range could only distinguish the $2.00 \mathrm{M}$. type at a distance of about $40 \mathrm{~cm}$. This indicated some slight improvement in vision. The tension was normal.

The left eye (the operated eye) showed a faint triargular outline of the area which was originally occupied by the rather dense whitish opacity. Below the centre of the cornea and slightly to the temporal side was a white macula about $2 \mathrm{~mm}$. in diameter the vestige of the large white area already referred to. The anterior chamber was normal in depth and there was no evidence that the linear sulcus of about $2 \mathrm{~mm}$. length, which could be seen at the temporal margin of the cornea, was a patulous opening into the anterior chamber. The tension was normal. It would have been diminished 
otherwise. There was no hernia of the choroid, and the heaping up of the episcleral tissue on the temporal side of this eye originally observed seemed to have been absorbed. The vision was $6 / 6$ without glasses and with glasses $6 / 6$ plus. Close vision as well as accommodation was normal. The patient could read the 0.50 M. type at $35 \mathrm{~cm}$. without difficulty.

On March 7 and 8, 192 $\tau$, the patient again presented herself for observation. The right eye at this time showed a decided squint. The cornea was entirely clear, the tension was normal, as were also the iris, lens, vitreous and eyegrounds. At the site of the original advancement operation on the external rectus there was a persistence of the heaping up of the conjunctiva and underlying tissue with a slight protrusion of the choroid-a slight hernia of the vascular coat. The vision was $6 / 30$ and could not be improved with lenses. This was a decrease in vision over the last observation.

The left eye was straight. The cornea showed a very small but dense white isolated scar, a trifle to the temporal side of the centre, but separated from the corneal margin by an area of clear and transparent cornea. Originally the opacity extended from its apex back to the corneal margin like an expanding fan. At the corneoscleral junction corresponding to the horizontal meridian there were two linear depressions, the smaller one above and the larger one below the horizontal meridian. These would suggest the possibility of fistulae, but the anterior chamber was of normal depth and there was no bulging forward of the iris although it could be seen more distinctly through these depressions than elsewhere. These corresponded to the incision made for the purpose of draining the corneal cyst. There was no evidence of the hernia of the choroid which was so prominent upon the initial observation. The tension was normal and the fundus which was readily seen with the ophthalmoscope was normal. The vision was $6 / 9$ without glasses and $6 / 6$ with glasses. Close vision and accommodation were normal in this eye, although originally almost nil. The fluctuations in vision in both eyes are worthy of comment.

In May, 1927, she was again examined in detail and showed no variation except in the matter of vision. Without glasses this was $6 / 60$ and $6 / 6$, but with correction under atropine cycloplegia it was $6 / 15$ and $6 / 6$.

In summarizing this case for purposes of discussion several features are outstanding. The matter of deep anchorage of the advancement is especially prominent. Many operators have felt that could their anchorages have been made sufficiently deep their advancements would have been more permanent. Here the anchorages were made exceptionally deep and yet some force was sufficient to overcome them and permit the original squint to return. 
Why a cyclitis did not supervene is also a matter for comment. The unusual development of a separation of the lamellae of the cornea is sufficiently rare to afford considerable discussion. The result following an original method of surgery was highly gratifying to the surgeon, but the fact that there was a physiological variation in the vision of the unoperated as well as the operated eye must not be overlooked. There was likewise a degree of spontaneous absorption of the steamy area in the cornea of the unoperated eye.

\section{A CASE OF SYMPATHETIC OPHTHALMITIS}

BY

\section{GEORGE YOUNG \\ COLCHESTER}

HERETOFORE ophthalmic surgeons have opposed the enucleation of the first eye, once the second has become affected by sympathetic ophthalmitis, if any degree of vision remains in the first eye. Experience derived from tragic lessons in the past had established this rule. The latest books of reference quote it. More recent summaries remind us of it again.(1) But when the slit-lamp enabled us to recognize much more recent signs in the second eye than had been possible previously, enthusiasm voiced the notion that it might be advisable to enucleate the first eye as soon as we saw those signs. On such occasions my mind rose in rebellion, for I believed in the old rule so firmly as to fancy that if the slit-lamp were to lead us into even considering such a step it were better to be on guard against actually taking it. Moreover, now that we could detect the very first signs in the second eye, we should be sooner warned; while had we failed to recognize these initial signs we might have removed the first eye judging the second to be still within the period of safety from sympathetic ophthalmitis.

Then this case came along. Perhaps to remind me that tempora mutantur. Its course gives food for reflection; and though it does not presume to overthrow, yet it challenges the old maxim. For it might actually prove correct to remove the first eye, vision or no vision, even after the earliest signs have appeared in the second eye.

T.H., aged 72 years, a ship's stoker, had a lubricating gauge glass burst and cut his left eye through the cornea and ciliary body in the upper outer quadrant on August 6, 1926. He was under the care of Mr. J. A. Valentine at the Portsmouth Eye Hospital till the end of August, when he returned home and presented himself at the Out-patient Department of the Essex County Hospital with a letter from my colleague asking me 